
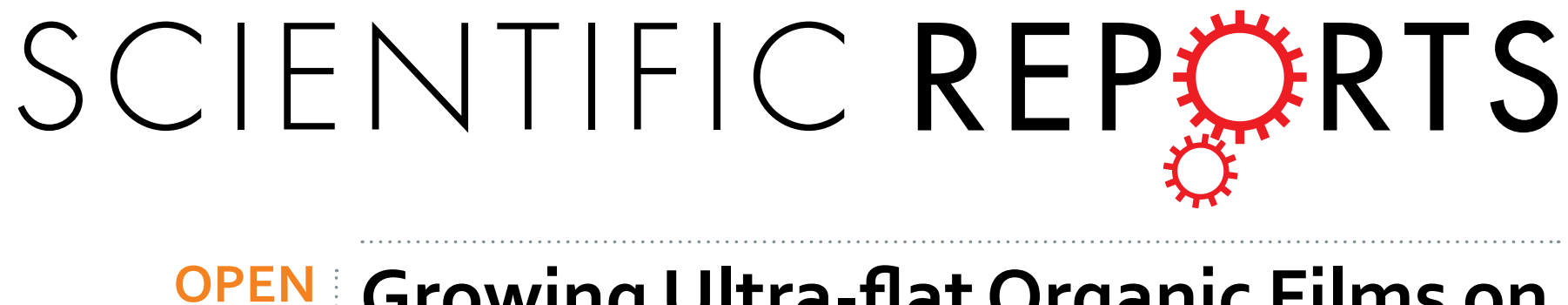

\title{
Growing Ultra-flat Organic Films on Graphene with a Face-on Stacking via Moderate Molecule-Substrate \\ Interaction
}

Received: 08 March 2016

Accepted: 10 June 2016

Published: 30 June 2016

\author{
Ti Wang, Tika R. Kafle, Bhupal Kattel, Qingfeng Liu, Judy Wu \& Wai-Lun Chan
}

The electronic properties of small molecule organic crystals depend heavily on the molecular orientation. For multi-layer organic photovoltaics, it is desirable for the molecules to have a face-on orientation in order to enhance the out-of-plane transport properties. However, it is challenging to grow well-ordered and smooth films with a face-on stacking on conventional substrates such as metals and oxides. In this work, metal-phthalocyanine molecules is used as a model system to demonstrate that two-dimensional crystals such as graphene can serve as a template for growing high quality, ultra-flat organic films with a face-on orientation. Furthermore, the molecule-substrate interaction is varied systematically from strong to weak interaction regime with the interaction strength characterized by ultrafast electron transfer measurements. We find that in order to achieve the optimum orientation and morphology, the molecule-substrate interaction needs to be strong enough to ensure a face-on stacking while it needs to be weak enough to avoid film roughening.

Organic photovoltaics (OPV) is one of the contenders for next generation solar cells due to its low cost, flexibility, light weight and ease of fabrication ${ }^{1-3}$. Most organic semiconductors are anisotropic. As a result, the relative orientations among different molecules and between molecules and the substrate play a decisive role in determining the electronic properties and hence the efficiency of the $\mathrm{OPV}^{4-8}$. In small molecule organic semiconductors, charge transport primarily occurs in the $\pi$-stacking direction because of the stronger electronic coupling ${ }^{9,10}$. Moreover, the electron transfer rate at heterostructure interfaces depends on the relative orientation between the donor and acceptor molecules. For example, at the copper phthalocyanine $(\mathrm{CuPc}) /$ fullerene $\left(\mathrm{C}_{60}\right)$ interface, it is found that the electron transfer rate from face-on $\mathrm{CuPc}_{\mathrm{P}} \mathrm{C}_{60}$ is four times larger than that from edge-on $\mathrm{CuPc}$ to $\mathrm{C}_{60}{ }^{5}$. In OPV applications, because electrons move along the device's out-of-plane direction, it is desirable to have the $\pi$-stacking direction parallel to the surface normal direction ${ }^{6}$. For most planar organic molecules, it means that the molecular plane is needed to be parallel to the molecule-substrate interface (face-on orientation) in order to attain efficient charge transport.

The orientation of small planar molecules on a substrate depends on the interplay between the molecule-substrate and molecule-molecule interactions ${ }^{11-13}$. For weak molecule-substrate interaction (e.g. molecules deposited on $\mathrm{SiO}_{2}$ ), the molecule-molecule interaction dominates and molecules tend to stand up with the $\pi$-stacking direction lying parallel to the interface. Although molecules can be grown in a layer-by-layer fashion with a smooth film morphology ${ }^{14,15}$, the out-of-plane electronic transport is slow. This edge-on orientation favors applications such as organic field effect transistors ${ }^{8,15}$, but it is undesirable for photovoltaics and other opto-electronic applications. On the other hand, for a strong molecule-substrate interaction (e.g. molecules deposited on metal substrates), the molecules have a face-on stacking ${ }^{16-19}$. However, the strong molecule-substrate interaction often results in a metastable crystal structure. Beyond a few monolayers, the film will relax by forming mounds on the surface. In some cases, the molecules will even switch to a standing-up orientation ${ }^{20,21}$. Indeed, it is a challenge to grow well-ordered and smooth organic films with a face-on orientation on conventional metal and oxide substrates ${ }^{22}$. Recently, it is demonstrated that one can circumvent this limitation by using substrate with an "intermediate" molecule-substrate interaction. For example, Si substrate is doped degenerately to reduce the molecule-substrate interaction such that thick face-on oriented films can be grown without significant

Department of Physics and Astronomy, University of Kansas, Lawrence, KS 66045, USA. Correspondence and requests for materials should be addressed toT.W. (email: wangti@ku.edu) orW.-L.C. (email: wlchan@ku.edu) 
surface roughening ${ }^{16}$. A few studies show that graphene is a good template for growing uniform organic films with a face-on stacking ${ }^{23-26}$. However, these studies mainly focus on the structural and electrical characterization of the organic layer grown on graphene. It is unclear how specifically the film morphology depends on the molecule-substrate interaction and why molecular crystals grown on graphene have better morphology compared to that grown on metals ${ }^{21}$ even though both has a face-on orientation.

In this work, $\mathrm{ZnPc}$ molecules are used as a model system to investigate the relationship between the morphology, molecular orientation and interfacial electronic properties by systematically varying the molecule-substrate interaction from strong to weak interaction regime. A previous comprehensive study of CuPc grown on graphene ${ }^{24}$ has shown that the morphology and molecule orientation can depend on the film thickness and the growth temperature. In this work, we will fix these other parameters to investigate the effect of the substrate on the film properties. A series of substrates including $\mathrm{Cu}, \mathrm{Au}$, graphite, graphene and $\mathrm{SiO}_{2} / \mathrm{Si}$, were selected to provide progressively weaker molecule-substrate interactions. Compared to previous works which mainly focus on the orientation of molecules on different substrates, we investigate how the initial molecule-substrate interaction would eventually affect the film morphology and roughness when the film becomes thicker. The film morphology and roughness, on top of the molecular orientation, can significantly affect the transport properties and device performance. The orientation of the molecules was determined by the work function of the film measured with ultraviolet photoemission spectroscopy (UPS) ${ }^{7,27}$, while the surface morphology was characterized using atomic force microscopy (AFM). Compared to the $\mathrm{Cu}$ and $\mathrm{Au}$, $\mathrm{ZnPc}$ thin films on the HOPG and graphene show a smoother morphology because the weaker molecule-substrate interaction in the latter allows the films to be grown with a more relaxed structure. However, the presence of step-edges on HOPG surfaces induces preferential nucleation of $\mathrm{ZnPc}$ islands at step-edges, resulting in a non-uniform film coverage. This issue can be addressed by using graphene since its atomic flat surface enables the formation of ultra-smooth ZnPc films with uniform coverage.

Furthermore, the interfacial charge transfer from the molecule to the substrate, which reflects the interfacial electronic coupling, is measured by femtosecond ( $\mathrm{fs}$ ) time-resolved photoemission spectroscopy (2PPE). The stronger molecule-substrate interaction at the $\mathrm{ZnPc} /$ metal interface compared to that at $\mathrm{ZnPc}$ graphite and $\mathrm{ZnPc/}$ graphene interfaces results in a faster charge transfer rate and a spatially larger interaction range. Among all the substrates studied, we find that graphene, which has a moderate interaction with the organic molecules, enables molecules to be grown with face-on orientation while avoiding significant structural relaxation as the film grows thicker.

\section{Results and Discussion}

Surface Morphology. All the metal substrates are cleaned in ultrahigh vacuum (UHV) environment using standard sputter and annealing procedures (see Method section). In the case of graphite, the surface is cleaved before subsequent annealing and deposition. The AFM images of the bare substrate are shown in Fig. 1. On all these substrates ( $\mathrm{Au}, \mathrm{Cu}, \mathrm{HOPG})$, the surface is cleaned and has a small initial rms roughness $(<0.7 \mathrm{~nm})$. Stepedges on these surfaces can be seen in the AFM images. For the graphene on $\mathrm{SiO}_{2}$ sample, the surface appears to have some wrinkles on the surface. It is known that a single layer of graphene can form wrinkles and ripples on its supporting substrate ${ }^{28}$. The small grain size found in CVD graphene ${ }^{29}$ may also result in these initial roughness. A discussion on the origin of this roughness is out of the scope of this work, but we note that the rms roughness of the graphene substrate is still small $(0.74 \mathrm{~nm})$.

Figure 2 shows the morphology measured by AFM for $10 \mathrm{~nm} \mathrm{ZnPc}$ films deposited on various substrates. Images with a larger scan size are presented in the Supplementary information, which show that the morphology is similar at a larger length scale. Similar morphology is observed on different areas of the samples. Moreover, a thickness of $10 \mathrm{~nm}$ is chosen because the effects of the substrate on the morphology are clearly manifested at this thickness. At larger thicknesses, other effects such as molecule-molecule interactions or grain coalescence may start to couple to the substrate-effect to affect the film morphology. For example, a recent work ${ }^{24}$ on $\mathrm{CuPc}$ films on graphene has found that, at room temperature, the molecules have a face-on orientation at a film thickness of $20 \mathrm{~nm}$, but some molecules switch to an edge-on orientation when the film thickness increases to $50 \mathrm{~nm}$. The switching of the molecular orientation suggest that molecule-molecule interaction dominates at larger thicknesses. Considering this work focuses primarily on revealing how the film morphology depends on the substrate-molecule interaction, a smaller thickness of $10 \mathrm{~nm}$ was selected to avoid the complication of molecule-molecule interaction.

For the film deposited on $\mathrm{Cu}(001)$ (Fig. 2a), many small islands are formed on the surface. The $\mathrm{ZnPc}$ film grown on $\mathrm{Au}(111)$ surface shows a clear increase in grain size (Fig. 2b). Figure 3 shows the phase images obtained from the same measurement shown in Fig. 2a,b. The $\mathrm{ZnPc} / \mathrm{Cu}$ shows a much stronger phase contrast than the $\mathrm{ZnPc} / \mathrm{Au}$ (Fig. 3). The phase contrast is commonly originated from a variation of surface composition or surface adhesion. The correlation between regions with a lower surface height and regions with a smaller phase angle (e.g. areas enclosed by red circles in Figs $2 \mathrm{a}$ and $3 \mathrm{a}$ ) indicates that part of the $\mathrm{Cu}$ surface is exposed. In contrast, the phase-image of $\mathrm{ZnPc} / \mathrm{Au}$ shows a more uniform intensity indicating that the $\mathrm{ZnPc}$ on $\mathrm{Au}$ has a complete coverage.

A number of studies have been devoted to investigate the interaction between small molecules and metal surfaces $^{18,19,30-32}$. It is known that organic molecules interact more strongly with $\mathrm{Cu}$ than with Au. For example, a recent study shows that when $\mathrm{CuPc}$ molecules are deposited on $\mathrm{Cu}$, commensurate islands are formed at monolayer coverage, indicating that molecules are chemisorbed on the surface. For Au surfaces, the CuPc molecules are physisorbed and behave as a two-dimensional gas, which allows the formation of a uniform wetting layer ${ }^{18}$. The stronger molecule-substrate interactions between $\mathrm{ZnPc}$ and $\mathrm{Cu}$ can reduce the mobility of $\mathrm{ZnPc}$ molecules on the $\mathrm{Cu}$ surface, which explains the smaller feature size and the discontinuity observed in the $\mathrm{ZnPc} / \mathrm{Cu}$ samples. Furthermore, our previous study on $\mathrm{ZnPc} / \mathrm{Au}$ shows that the film has a layer-by-layer growth mode at film thickness $<5 \mathrm{~nm}^{33}$, but the island morphology observed in Fig. $2 \mathrm{~b}$ develops only when the film becomes thicker. The 
(a)

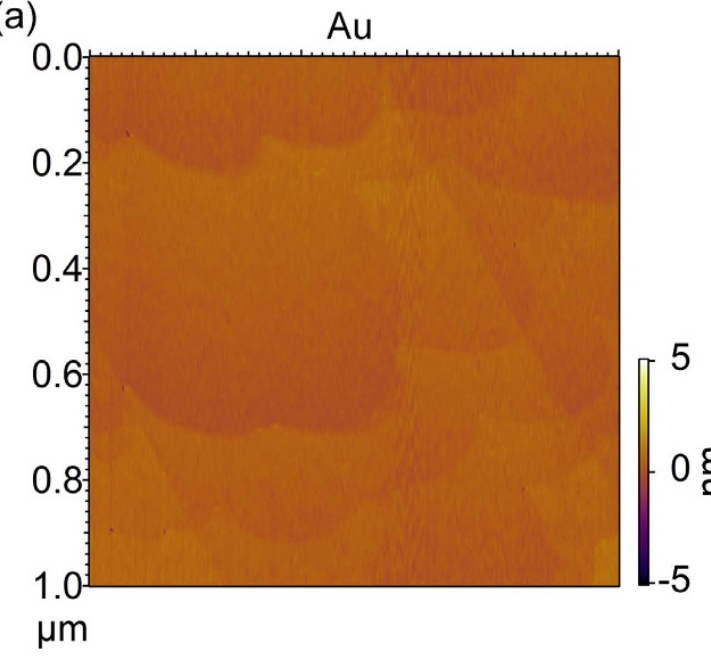

(c)

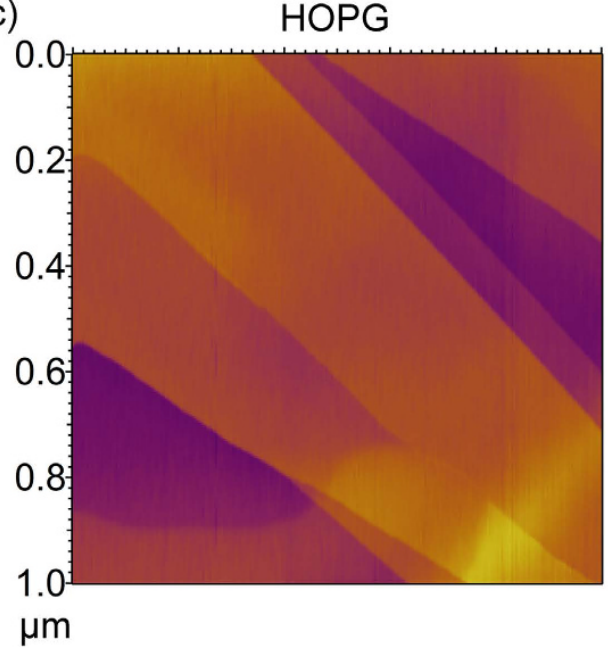

(b)

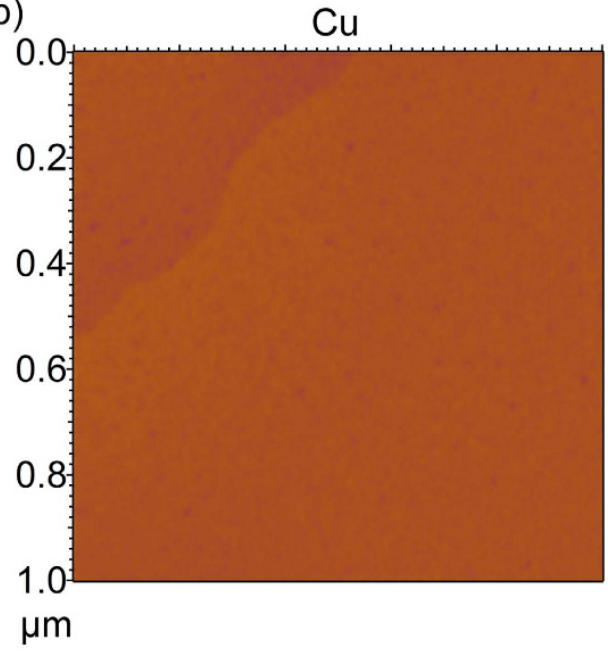

(d)

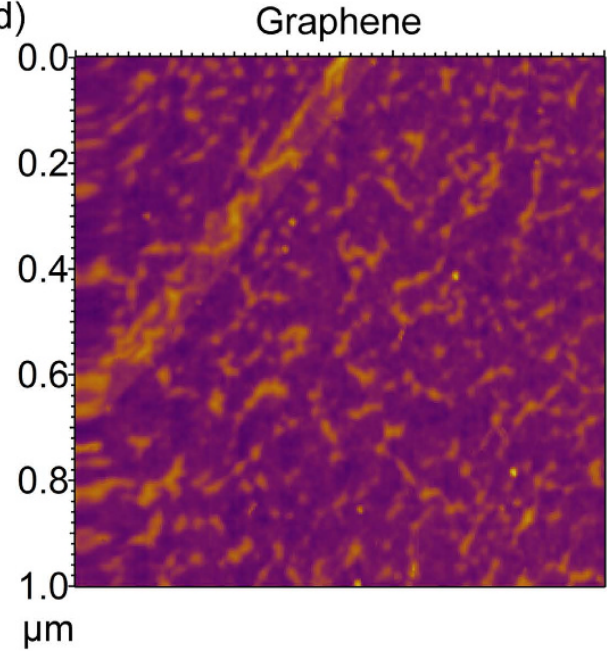

Figure 1. AFM images of the (a) $\mathrm{Au},(\mathbf{b}) \mathrm{Cu},(\mathbf{c}) \mathrm{HOPG}$, and (d) graphene substrates before $\mathrm{ZnPc}$ deposition.

AFM images of the $2 \mathrm{~nm} \mathrm{ZnPc} \mathrm{film} \mathrm{on} \mathrm{Au} \mathrm{are} \mathrm{shown} \mathrm{in} \mathrm{the} \mathrm{Supplementary} \mathrm{information.} \mathrm{It} \mathrm{shows} \mathrm{that} \mathrm{most} \mathrm{parts}$ of the film are smooth with some small islands starting to nucleate on the surface. This kind of growth mode is typically known as the Stranski-Krastanov (SK) growth ${ }^{34}$. The formation of islands is usually driven by the relaxation of the film stress. In our case, the $\mathrm{ZnPc}$ - Au interaction can result in a $\mathrm{ZnPc}$ crystal that has slightly different lattice parameters compared to that of the equilibrium structure (i.e. the crystal is strained). The formation of islands at a later stage is evidently driven by structural relaxation.

The films grown on $\mathrm{Cu}$ and $\mathrm{Au}$ either show an incomplete coverage or an island growth morphology at large film thicknesses. In order to grow ultra-smooth and well-ordered films with uniform coverage, further weakening of the molecule-substrate interaction is necessary. Substrates such as graphite and graphene interact with ZnPc via weaker van der Waal's interaction. However, the molecule-substrate interaction should still be strong enough to ensure a face-on stacking ${ }^{35-37}$. The surface morphology of ZnPc films grown on HOPG is shown in Fig. 2c. As seen in Fig. 2c, the top of the ZnPc grains are atomically flat, but some deep gaps exist between grains. Comparing Figs $2 c$ and $1 c$, the shape of the grains correlates with the shape of the atomic terraces on the bare graphite surface. Therefore, the formation of these deep gaps is apparently related to the step edges on the graphite surface. For example, a recent work on $\mathrm{ZnPc}$ on $\mathrm{Si}(111)$-Boron surface shows that the $\mathrm{ZnPc}$ nucleates preferentially on the step-edges of $\mathrm{Si}(111)$ surfaces $^{16}$.

Further improvement in the film morphology requires elimination of the surface step-edges. This motivates us to use graphene as a template for $\mathrm{ZnPc}$ growth, which does not contain any step edge on the surface. Fig. $2 \mathrm{~d}$ shows the AFM image of $10 \mathrm{~nm} \mathrm{ZnPc} \mathrm{deposited} \mathrm{on} \mathrm{graphene.} \mathrm{The} \mathrm{ZnPc} \mathrm{film} \mathrm{covers} \mathrm{the} \mathrm{substrate} \mathrm{uniformly} \mathrm{with} \mathrm{a}$ low root mean square (RMS) roughness $(2.47 \pm 0.28 \mathrm{~nm})$. It is not clear whether the small-sized features observed correspond merely to surface roughness or to a film with a small grain size. However, the lateral size of these features is similar to those observed on the bare graphene surface (Fig. 1d). We expect that the quality of the film would be further improved if the graphene does not have those wrinkles observed in Fig. 1d. Note that the surfaces of the $\mathrm{ZnPc}$ grains deposited on both graphene and graphite are smooth, but some discontinuities are found in the film on graphite due to the presence of step edges on the bare graphite surface. Based on this observation, it 

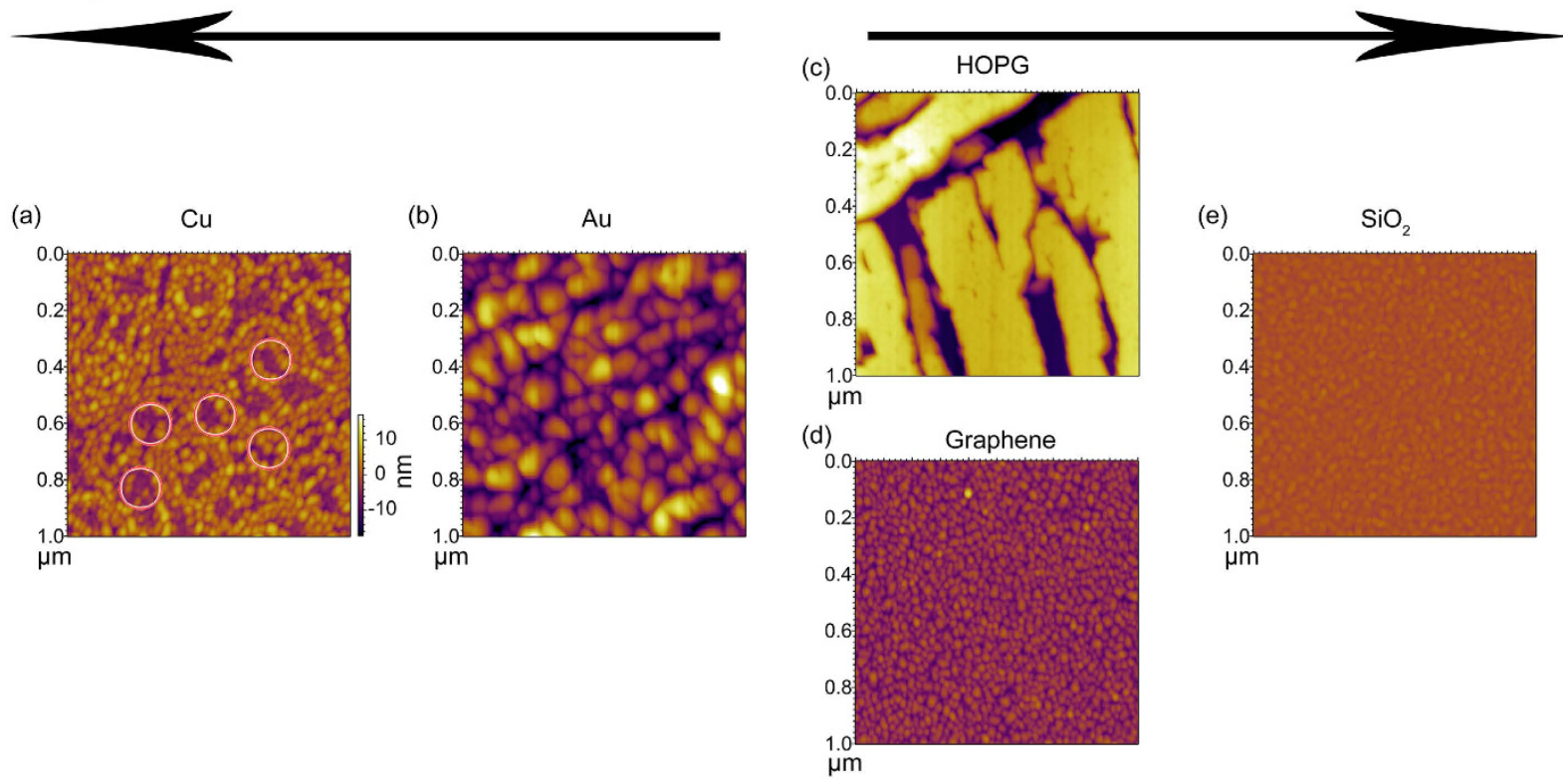

Figure 2. AFM images of $10 \mathrm{~nm} \mathrm{ZnPc}$ thin films deposited on (a) $\mathrm{Cu},(\mathbf{b}) \mathrm{Au},(\mathbf{c}) \mathrm{HOPG}$, (d) graphene and (e) $\mathrm{SiO}_{2}$. For comparison, the same color scale is used for all the images.

(a)

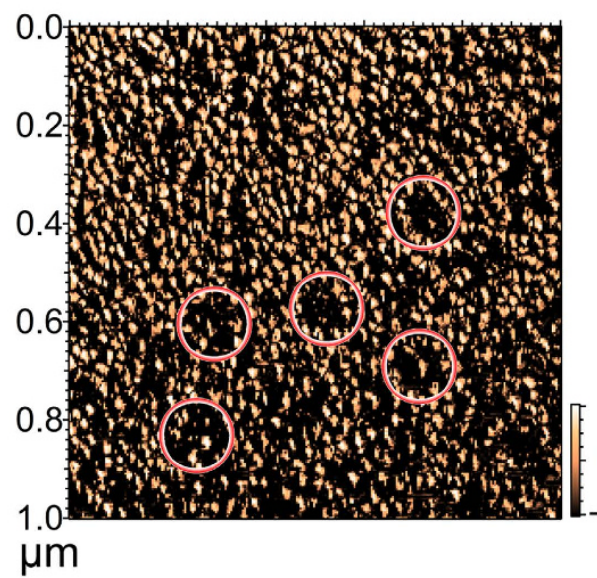

(b)

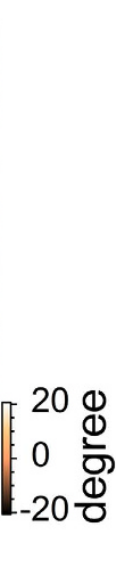

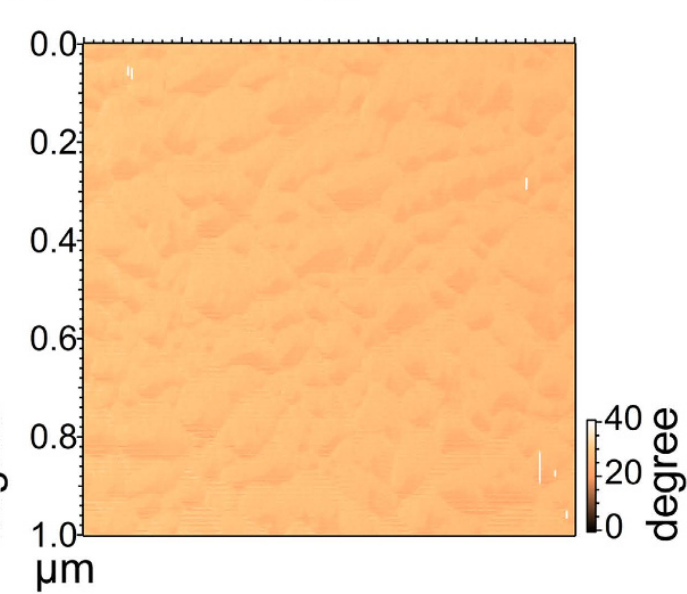

Figure 3. The AFM phase images of $\mathrm{ZnPc}$ on (a) $\mathrm{Cu}$ and (b) Au obtained from the same regions shown in Fig. 1a,b, respectively. The red circles in (a) are the same positions presented in Fig. 2a, in which the phase angle has consistently smaller value compared to that in other regions.

is expected that the morphology of $\mathrm{ZnPc}$ films grown on multi-layer graphene should be similar to that on single layer graphene if the top graphene layer is continuous.

One may further reduce the molecule-substrate interaction by using substrates such as $\mathrm{SiO}_{2} / \mathrm{Si}$. However, it is known that because the molecule-molecule interaction is now stronger than the molecule-substrate interaction, the molecules will have an edge-on (or standing-up) orientation with the fast transport direction parallel to the surface. Indeed, these films usually show extremely smooth morphology because the nucleation of a new layer is a slow process and the growth proceeds with a layer-by-layer mode. Fig. 2e shows the morphology of ZnPc films on $\mathrm{SiO}_{2} / \mathrm{Si}$ with the RMS roughness of $0.78 \pm 0.03 \mathrm{~nm}$, which is less than the height $(\sim 1.2 \mathrm{~nm}$ for the edge-on orientation) of the molecule.

Molecular orientation. As mentioned earlier, electronic properties of organic films depend heavily on the orientation of the molecules. Here, the molecular orientation is determined by UPS. Previous works have shown that the ionization potential of molecular crystals depends on the orientations of molecules with respect to the substrates. For pi-conjugated small molecules, films with face-on molecules typically have an ionization potential 

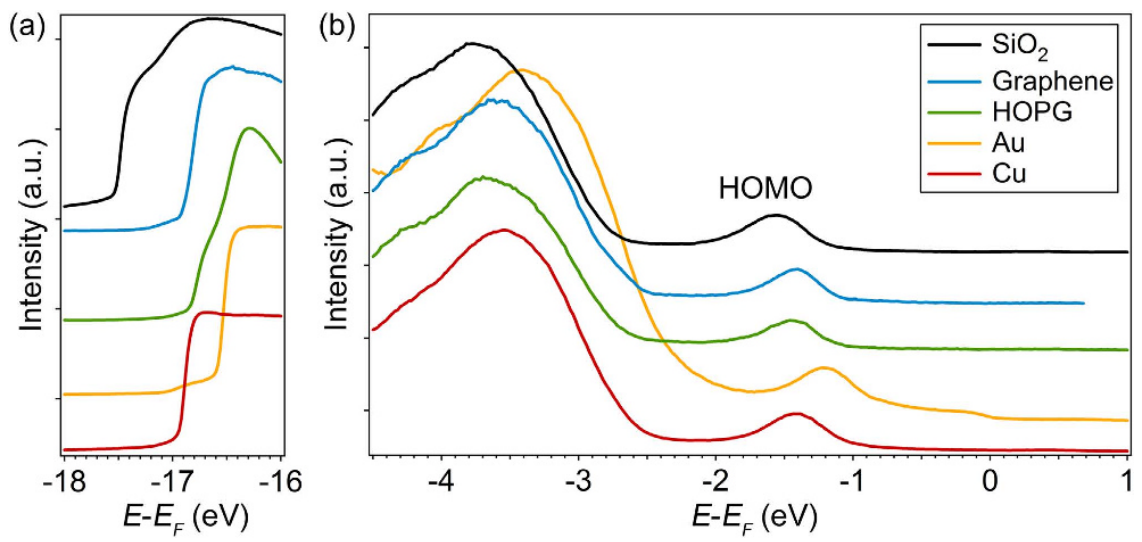

Figure 4. UPS spectra of $10 \mathrm{~nm} \mathrm{ZnPc}$ thin films on $\mathrm{SiO}_{2}$, graphene, $\mathrm{HOPG}, \mathrm{Au}$ and $\mathrm{Cu}$. The secondary electron cutoff and the HOMO are shown in (a) and (b) respectively. The UPS spectra are collected with the He-I emission line with photon energy of $21.22 \mathrm{eV}$.

$\sim 0.5 \mathrm{eV}$ larger than that for films with edge-on molecules ${ }^{7,27}$. Therefore, the ionization potentials of $\mathrm{ZnPc}$ can be used to determine the orientations of the molecules. Figure 4 shows the UPS spectra of $10 \mathrm{~nm}$-thick ZnPc films deposited on various substrates. The region near the secondary electron cutoff (SECO) and the highest occupied molecular orbital (HOMO) are shown in panel (a) and (b) respectively. The ionization potentials can be calculated from the position of the SECO and the HOMO edge, which are $5.24 \mathrm{eV}, 5.23 \mathrm{eV}, 5.27 \mathrm{eV}$ and $5.28 \mathrm{eV}$ for $10 \mathrm{~nm}$ $\mathrm{ZnPc}$ films on $\mathrm{Cu}, \mathrm{Au}, \mathrm{HOPG}$ and graphene respectively. On the other hand, the ionization potential decreases to $4.82 \mathrm{eV}$ for the $\mathrm{ZnPc}$ films deposited on $\mathrm{SiO}_{2} / \mathrm{Si}$. It is known that molecules deposited on $\mathrm{SiO}_{2}$ have an edge-on orientation because of the weak substrate-molecule interaction ${ }^{14,15}$. The ionization potential of $\mathrm{ZnPc}$ films found on other substrates is about $0.4-0.5 \mathrm{eV}$ larger, which indicates that the molecules adopt a face-on structure on $\mathrm{Cu}, \mathrm{Au}, \mathrm{HOPG}$ and graphene. The measured ionization potential is consistent with previous measurements on $\mathrm{CuPc}$ thin films ${ }^{27}$, in which $\mathrm{CuPc}$ molecules with face-on and edge-on orientations have an ionization potential of $5.15 \mathrm{eV}$ and $4.75 \mathrm{eV}$ respectively. The position of the SECO for the edge-on domains would appear at energy $\sim 0.4 \mathrm{eV}$ lower compared to that of the face-on domains. The sharpness of the SECO and the weak photoemission intensity below the cutoff energy found in $\mathrm{ZnPc}$ /graphene indicates that most of the molecules have a face-on orientation. This agrees with a comprehensive X-ray study done by Xiao et al. ${ }^{24}$, which shows that CuPc on graphene has a face-on orientation for film thicknesses up to $20 \mathrm{~nm}$.

To further support the UPS results, low-energy electron diffraction (LEED) is done for $10 \mathrm{~nm} \mathrm{ZnPc}$ on $\mathrm{Au}$ and HOPG. The diffraction patterns are shown in the supplementary information, which show that the ZnPc molecules on Au and HOPG have a face-on orientation and consistent with the orientation determined using the ionization potential. Moreover, similar UPS and LEED results can be obtained on different areas of the surfaces, which indicates that the same molecular orientation is found across the film.

In our earlier discussion, it is mentioned that the $\mathrm{ZnPc}$ film on $\mathrm{Cu}$ does not fully cover the surface, which is also reflected in our surface-sensitive UPS measurements. Figure $5 \mathrm{a}$ shows the UPS spectra of $\mathrm{Cu}(001), 1 \mathrm{~nm}$, $3 \mathrm{~nm}$ and $10 \mathrm{~nm}$-thick $\mathrm{ZnPc}$ on $\mathrm{Cu}(001)$. Since UPS is a surface sensitive technique, the spectra represent signal originated from the film surface. Copper has a d-band peak located between $2 \mathrm{eV}$ and $4 \mathrm{eV}$ below the Fermi level. The lower energy edge (enclosed by the dashed rectangle) of this d-band peak is clearly observed for the $1 \mathrm{~nm}$ and the $3 \mathrm{~nm} \mathrm{ZnPc} \mathrm{films.} \mathrm{This} \mathrm{edge} \mathrm{can} \mathrm{still} \mathrm{be} \mathrm{observed} \mathrm{in} \mathrm{the} 10 \mathrm{~nm}$-thick $\mathrm{ZnPc}$ film, even though the intensity is much reduced. Therefore, we conclude that a portion of the $\mathrm{Cu}$ surface is still exposed for films as thick as $10 \mathrm{~nm}$, which is consistent to the AFM measurement. For comparison, the corresponding spectra for ZnPc films grown on $\mathrm{Au}(111)$ are shown in Fig. 5b. Similar to $\mathrm{Cu}$, Au has a d-band peak located at around 2-6 eV below the Fermi level. Some of the feature from the d-band peak can be observed in the spectrum of the $1 \mathrm{~nm}$ film (dashed rectangle). However, for larger thicknesses, the spectra resemble to that of the thick $\mathrm{ZnPc}$ film. As shown in Fig. 5b, the $3 \mathrm{~nm}$ spectrum is almost identical to the $10 \mathrm{~nm}$ spectrum. This is consistent to our earlier interpretation that $\mathrm{ZnPc}$ forms a complete wetting layer on the Au surface before transitioning to an island growth mode.

Electronic coupling at the molecule-substrate interface. Molecular orientation affects the dynamical charge transfer rate at the interface ${ }^{5,6}$ because the electronic-coupling between the donor and acceptor molecules depends strongly on their relative orientations. Similarly, a stronger electronic coupling between the substrate and interface should result in a faster interfacial charge transfer rate. Here, we characterize the interfacial charge transfer rate by using $2 \mathrm{PPE}$ spectroscopy. We have used this technique previously to characterize the interfacial charge transfer rate for $\mathrm{ZnPc}$ molecules deposited on graphene ${ }^{38}$. We note that other similar techniques such as resonant photoemission spectroscopy have also been used to study charge transfer across molecule/metal interfaces $^{39}$. The details of our ultrafast experiment can be found in refs 33,38. Briefly, since 2PPE is surface sensitive, the lifetime of the optically-excited exciton in ultrathin ZnPc layer deposited on various substrates is determined by the charge transfer rate from the molecules to the substrate. Figure 6 shows the integrated photoemission intensity originated from the optically-excited singlet exciton of ZnPc deposited on Au, HOPG and graphene. The photoemission intensity can be interpreted as the population of the singlet exciton located near the surface. 
(a)

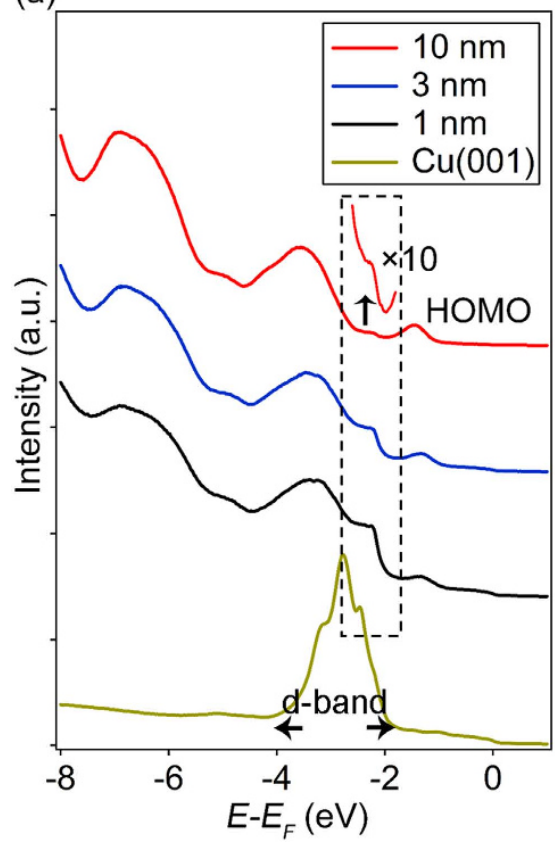

(b)

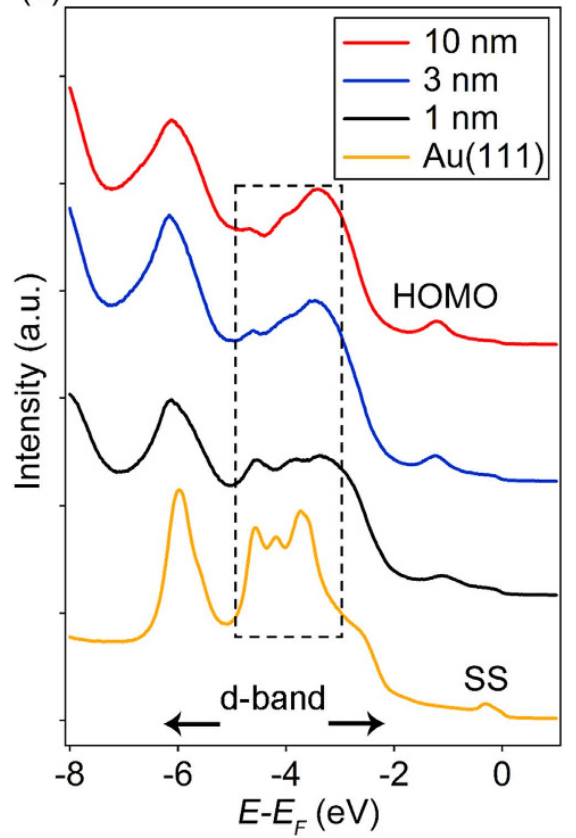

Figure 5. (a) UPS spectra of $\mathrm{Cu}(001), 1,3$ and $10 \mathrm{~nm} \mathrm{ZnPc}$ thin films on $\mathrm{Cu}$. (b) UPS spectra of $\mathrm{Au}(111), 1,3$ and $10 \mathrm{~nm} \mathrm{ZnPc}$ thin films on Au. The "SS" label represented the surface state of Au.

The results for three different film thicknesses: $0.5 \mathrm{~nm}, 1 \mathrm{~nm}$ and $10 \mathrm{~nm}$ are shown. The dynamics observed for the $10 \mathrm{~nm}$ samples on various substrates are almost identical. This is because excitons near the film surface cannot interact with the substrate at this short time-scale and the dynamics represents the intrinsic dynamics of the $\mathrm{ZnPc}$ thin film.

For the 0.5 and $1 \mathrm{~nm}$ samples, the photoemission intensity shows a more rapid decay. The initial ultrafast decay of the intensity is attributed to the charge transfer from $\mathrm{ZnPc}$ thin films to the substrates. After the first few hundreds fs, the intensity decreases at a much slower rate. This is attributed to the relaxation of excited electrons to lower energy traps, which can slower down the electron transfer. The initial decay is fitted with an exponential function (solid lines) convoluted with the finite temporal-width of the laser pulses. The decay times (the inverse of the decay rate) obtained from the fit are summarized in Table 1.

Two main observations can be made. First, the decay times are shorter for Au and progressively become larger for graphite and graphene. In our previous work, we find that the band-alignment at the molecule/substrate interface is similar for graphite and graphene. In both cases, the singlet level is about $0.3-0.4 \mathrm{eV}$ higher than the Fermi level of the substrate ${ }^{38}$. For $\mathrm{ZnPc} / \mathrm{Au}$, the singlet level of $\mathrm{ZnPc}$ is located at $0.65 \mathrm{eV}$ above the Fermi level of $\mathrm{Au}^{38}$. Because of the relative small reorganization energy $(0.1-0.2 \mathrm{eV})$ of $\mathrm{ZnPc}$ molecules ${ }^{40,41}$, we expect that the electrons will transfer to states in the substrate that have similar energy to the molecular state ${ }^{42}$. In all these substrates, since unoccupied states are available at all energies above the Fermi level, electrons can be transferred directly to states with energies around the singlet level. Therefore, it is not expected that the actual energy alignment would significantly affect the transfer rate. On the other hand, the slower transfer rate for graphene compared to that for graphite can be explained by the reduced dimensionality (from 3D to 2D). It is known that the density of states near the Fermi level in graphene is reduced significantly compared to that of graphite ${ }^{43}$. The smaller number of acceptor states in graphene would result in the lower transfer rate even though the ZnPc/graphite and $\mathrm{ZnPc/}$ graphene should have similar interfacial structure. The even faster transfer rate to Au would indicate that the molecule-substrate interaction is stronger for $\mathrm{Au}$ as compared to that for graphite and graphene. The very fast charge transfer rate in $\mathrm{ZnPc} / \mathrm{Au}$ also agrees with previous core-hole clock photoemission spectroscopy studies on $\mathrm{CuPc} / \mathrm{Au}^{44}$. The stronger molecule/substrate interaction in $\mathrm{ZnPc} / \mathrm{Au}$ is in accord with the evolution of the surface morphology discussed earlier.

Second, the decay time for $\mathrm{ZnPc}$ on $\mathrm{Au}$ increases only slightly when the thickness increases from 0.5 to $1 \mathrm{~nm}$. On the other hand, the decay times for ZnPc on HOPG and graphene are almost doubled when the film thickness is increased from $0.5 \mathrm{~nm}$ to $1 \mathrm{~nm}$. This result implies that Au has a spatially larger molecule-substrate interaction range with $\mathrm{ZnPc}$ molecules as compared to that of graphite and graphene. Since the height of lying down $\mathrm{ZnPc}$ molecules is $\sim 0.32 \mathrm{~nm}^{45}$, the interaction range between $\mathrm{ZnPc}$ and Au can extend up to a few molecular layers. The electronic wavefunction for states originated from metal substrates often has a tail that extends above the surface $^{46}$, which would result in a long range interaction between $\mathrm{ZnPc}$ and Au. The larger interaction range would induce a crystal structure in $\mathrm{ZnPc}$ that is slightly deviated from the crystal structure for thicker films. This explains why the $\mathrm{ZnPc}$ on Au follows the classical SK-growth mode. When the initial wetting layer reaches a certain critical thickness, structural relaxation drives the nucleation of grains with a more stable structure. This results in the formation of islands at larger film thicknesses. 
(a)

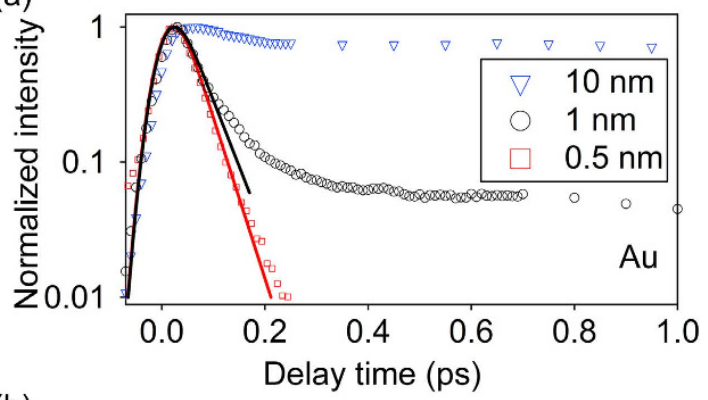

(b)

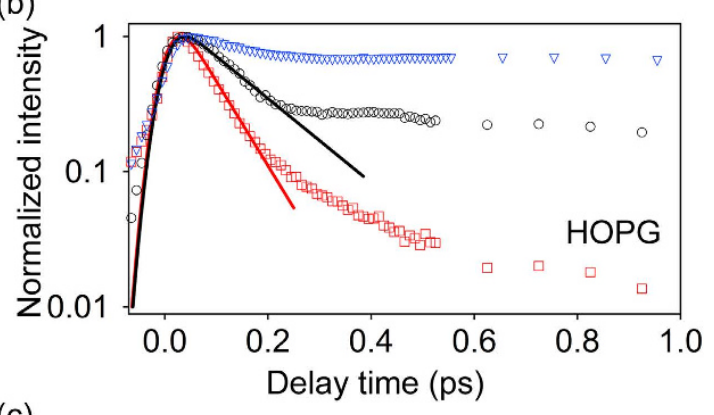

(c)

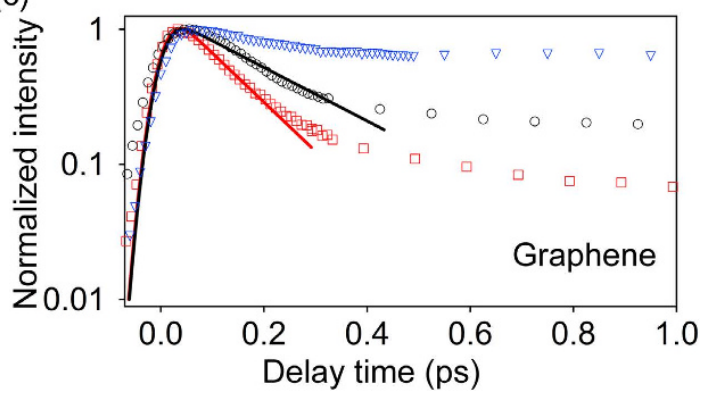

Figure 6. The integrated and normalized photoemission intensity of the $\mathrm{ZnPc}$ singlet state as a function of time for $0.5 \mathrm{~nm}, 1 \mathrm{~nm}$, and $10 \mathrm{~nm}$-thick ZnPc deposited on (a) Au, (b) HOPG and (c) graphene. The initial CT rate is fitted with an exponential function convoluted with the instrumental response function. The fits are shown as solid lines.

\begin{tabular}{|l|c|c|}
\hline Substrate & $\mathbf{0 . 5} \mathbf{n m}$ & $\mathbf{1} \mathbf{n m}$ \\
\hline $\mathrm{Au}$ & $40 \pm 10 \mathrm{fs}$ & $45 \mathrm{fs} \pm 10 \mathrm{fs}$ \\
\hline HOPG & $70 \pm 10 \mathrm{fs}$ & $140 \mathrm{fs} \pm 10 \mathrm{fs}$ \\
\hline Graphene & $140 \pm 20 \mathrm{fs}$ & $220 \mathrm{fs} \pm 20 \mathrm{fs}$ \\
\hline
\end{tabular}

Table 1. The decay times of the photoemission intensity for the $0.5 \mathrm{~nm}$ and the $1 \mathrm{~nm} \mathrm{ZnPc}$ films deposited on various substrates. These decay times are determined by fitting the experimental data shown Fig. 5 with an exponential decay function convoluted with the finite temporal width of the laser pulses.

Finally, we like to comment that the samples used to determine the charge transfer rate is either $0.5 \mathrm{~nm}$ or $1 \mathrm{~nm}$ thick, which should allow them to probe exclusively the molecule-substrate interaction. Any difference in the long-range structural order and the morphology of the $\mathrm{ZnPc}$ films as the film subsequently grown thicker cannot affect the measured charge transfer rate. Moreover, monolayer of planar metal-phthalocyanines has very similar orientation and crystal structure when they are deposited on $\mathrm{Au}(111)^{47}$ and HOPG surfaces ${ }^{48}$. Therefore, the difference in transfer rate is not likely to be caused by a difference in the molecular orientation.

\section{Conclusions}

In conclusion, by varying the molecule-substrate interaction that covers the strong $(\mathrm{Cu}, \mathrm{Au})$, intermediate (graphite, graphene) and weak $\left(\mathrm{SiO}_{2}\right)$ interaction regime, the change in the surface morphology and molecular orientation of $\mathrm{ZnPc}$ films deposited of these substrates has been investigated systematically. It has been found that in the intermediate interaction regime, the ZnPc molecules can maintain the face-on stacking, while the surface morphology is significantly improved as compared to those deposited on metal substrates of a strong molecule-substrate interaction. The variation of the molecule-substrate interaction is determined independently by measuring the electron transfer rate between the molecules and the substrate. On metal substrates, a stronger 
electronic coupling and a larger interaction range are found as compared to graphite and graphene. Our work illustrates the importance in controlling the molecule-substrate interaction in achieving a desirable morphology for the organic films. In this work, film thickness up to $10 \mathrm{~nm}$ is investigated, but it is expected that an initial smooth film morphology and the layer-by-layer growth mode observed in ZnPc on graphene should suppress film roughening and nucleation of grain with different orientations as the film grows thicker. Growing smooth and continuously organic films with a face-on stacking is important for photovoltaics applications. The current work shows that graphene and related 2D materials can be good templates for growing such organic films.

\section{Method}

Sample Preparation. $\quad \mathrm{ZnPc}$ thin films were deposited on $\mathrm{Cu}(001), \mathrm{Au}(111), \mathrm{HOPG}$, graphene and $\mathrm{SiO}_{2} /$ $\mathrm{Si}$ by thermal evaporation in an ultrahigh vacuum (UHV) chamber with base pressure $<5 \times 10^{-10}$ Torr. The $\mathrm{Cu}(001)$ and $\mathrm{Au}(111)$ single crystal surfaces were prepared by sputtering with $1 \mathrm{keV}$ Ar ions and annealing at $500^{\circ} \mathrm{C}$ repeatedly until a clean surface was obtained. For the HOPG substrate, the surface layer was peeled off with scotch tape to expose a fresh surface before it was introduced into the vacuum chamber. Single-layer graphene was either grown directly on $\mathrm{SiO}_{2} / \mathrm{Si}$ using $\mathrm{CVD}^{49}$ or transferred from $\mathrm{Cu}$ foil onto $\mathrm{SiO}_{2} / \mathrm{Si}$ using $\mathrm{PMMA}^{50}$. No significant difference was observed in the results obtained from the two different graphene. $\mathrm{The}_{\mathrm{SiO}} / \mathrm{Si}$ wafer was clean in methanol and acetone before it was introduced into the vacuum chamber. The HOPG, graphene and $\mathrm{SiO}_{2} / \mathrm{Si}$ samples were annealed in the UHV chamber at $400^{\circ} \mathrm{C}$ for 12 hours before deposition. The $\mathrm{ZnPc}$ molecules (Luminescence Technology, $>99 \%$ ) were vapor-deposited on different substrates at room temperature at a rate $\sim 0.8-1 \AA / \mathrm{min}$. Then, the sample was transferred under UHV environment to another chamber with a base pressure $<1 \times 10^{-10}$ Torr where UPS and time-resolved 2PPE experiments were performed.

Sample Characterization. The AFM was done with a Digital Instruments Multimode V atomic force microscope. Tapping-mode was used to avoid surface damages. The UPS experiment was performed with the He-I $(21.2 \mathrm{eV})$ line from a standard gas discharged lamp. For the ultrafast time-resolved measurements, the sample was excited by visible pump laser pulses with wavelength centered at $700 \mathrm{~nm}$. The pulses, which had duration of $\sim 25 \mathrm{fs}$, were generated from a non-collinear optical parametric amplifier NOPA (Orpheus- N-2H, Light Conversion). The excited electron was ionized by a time-delayed UV probe laser pulse (wavelength $\sim 280 \mathrm{~nm}$ ) which was generated by frequency-doubling the output of a second NOPA (Orpheus- N-3H, Light Conversion). Both NOPAs were pumped by a Yb:KGW regenerative amplifier running at a rate of $125 \mathrm{kHz}$ (Pharos $10 \mathrm{~W}$, Light Conversion). The kinetic energy of the photoelectron was determined by a hemispherical electron analyzer (SPECS Phoibos 100). Further details of the experimental setup can be found in refs 33 and 38 .

\section{References}

1. Roncali, J., Leriche, P. \& Blanchard, P. Molecular materials for organic photovoltaics: small is beautiful. Adv. Mater. 26, 3821-3838 (2014).

2. Graham, K. R. et al. Importance of the donor: fullerene intermolecular arrangement for high-efficiency organic photovoltaics. J. Amer. Chem. Soc. 136, 9608-9618 (2014).

3. Li, H. et al. Beyond fullerenes: design of nonfullerene acceptors for efficient organic photovoltaics. J. Amer. Chem. Soc. 136, 14589-14597 (2014).

4. Sai, N. et al. Understanding the interface dipole of copper phthalocyanine ( $\mathrm{CuPc}) / \mathrm{C} 60$ : theory and experiment. J. Phys. Chem. Lett. 3, 2173-2177 (2012).

5. Ayzner, A. L. et al. Ultrafast electron transfer at organic semiconductor interfaces: importance of molecular orientation. J. Phys. Chem. Lett. 6, 6-12 (2014).

6. Rand, B. P. et al. The impact of molecular orientation on the photovoltaic properties of a phthalocyanine/fullerene heterojunction. Adv. Funct. Mater. 22, 2987-2995 (2012).

7. Duhm, S. et al. Orientation-dependent ionization energies and interface dipoles in ordered molecular assemblies. Nat. Mater. 7, 326-332 (2008).

8. Zhang, Y. et al. Probing carrier transport and structure-property relationship of highly ordered organic semiconductors at the twodimensional limit. Phys. Rev. Lett. 116, 016602 (2016).

9. Cornil, J., Beljonne, D., Calbert, J. P. \& Brédas, J. L. Interchain interactions in organic $\pi$-conjugated materials: impact on electronic structure, optical response, and charge transport. Adv. Mater. 13, 1053-1067 (2001).

10. Curtis, M. D., Cao, J. \& Kampf, J. W. Solid-state packing of conjugated oligomers: From $\pi$-stacks to the herringbone structure. J. Amer. Chem. Soc. 126, 4318-4328 (2004).

11. Barth, J. V. Molecular architectonic on metal surfaces. Annu. Rev. Phys. Chem. 58, 375-407 (2007).

12. Götzen, J., Käfer, D., Wöll, C. \& Witte, G. Growth and structure of pentacene films on graphite: Weak adhesion as a key for epitaxial film growth. Phys. Rev. B. 81, 085440 (2010).

13. Thayer, G. E. et al. Role of surface electronic structure in thin film molecular ordering. Phys. Rev. Lett. 95, 256106 (2005).

14. Verlaak, S. et al. Nucleation of organic semiconductors on inert substrates. Phys. Rev. B. 68, 195409 (2003).

15. Li, L. et al. An ultra closely $\pi$-stacked organic semiconductor for high performance field-effect transistors. Adv. Mater. 19, 2613-2617 (2007).

16. Wagner, S. R., Lunt, R. R. \& Zhang, P. Anisotropic crystalline organic step-flow growth on deactivated Si surfaces. Phys. Rev. Lett. 110, 086107 (2013).

17. Wagner, S. R. et al. Growth of metal phthalocyanine on deactivated semiconducting surfaces steered by selective orbital coupling. Phys. Rev. Lett. 115, 096101 (2015).

18. Stadtmüller, B., Kröger, I., Reinert, F. \& Kumpf, C. Submonolayer growth of CuPc on noble metal surfaces. Phys. Rev. B. 83, 085416 (2011).

19. Bobaru, S. C., Salomon, E., Layet, J.-M. \& Angot, T. Structural properties of iron phtalocyanines on Ag (111): from the submonolayer to monolayer range. J. Phys. Chem. C. 115, 5875-5879 (2011).

20. Käfer, D., Ruppel, L. \& Witte, G. Growth of pentacene on clean and modified gold surfaces. Phys. Rev. B. 75, 085309 (2007).

21. Zheng, Y. et al. Effect of molecule-substrate interaction on thin-film structures and molecular orientation of pentacene on silver and gold. Langmuir 23, 8336-8342 (2007).

22. Witte, G. \& Wöll, C. Growth of aromatic molecules on solid substrates for applications in organic electronics. J. Mater. Res. 19, 1889-1916 (2004). 
23. Mativetsky, J. M. et al. Face-on stacking and enhanced out-of-plane hole mobility in graphene-templated copper phthalocyanine. Chem. Commun. 50, 5319-5321 (2014).

24. Xiao, K. et al. Surface-induced orientation control of CuPc molecules for the epitaxial growth of highly ordered organic crystals on graphene. J. Amer. Chem. Soc. 135, 3680-3687 (2013).

25. McAfee, T. et al. Toward single-crystal hybrid-carbon electronics: impact of graphene substrate defect density on copper phthalocyanine film growth. Cryst. Growth Des. 14, 4394-4401 (2014).

26. Mao, H. Y. et al. Chemical vapor deposition graphene as structural template to control interfacial molecular orientation of chloroaluminium phthalocyanine. Appl. Phys. Lett. 99, 093301 (2011).

27. Chen, W. et al. Molecular orientation-dependent ionization potential of organic thin films. Chem. Mater. 20, 7017-7021 (2008).

28. Deng, S. \& Berry, V. Wrinkled, rippled and crumpled graphene: an overview of formation mechanism, electronic properties, and applications. Mater. Today, In Press (2015)

29. Huang, P. Y. et al. Grains and grain boundaries in single-layer graphene atomic patchwork quilts. Nature 469, 389-392 (2011).

30. Stadler, C. et al. Tuning intermolecular interaction in long-range-ordered submonolayer organic films. Nat. Phys. 5, 153-158 (2009).

31. Mannsfeld, S. C. B. \& Fritz, T. Understanding organic-inorganic heteroepitaxial growth of molecules on crystalline substrates: experiment and theory. Phys. Rev. B. 71, 235405 (2005).

32. Stadler, C. et al. Structural investigation of the adsorption of SnPc on $\mathrm{Ag}$ (111) using normal-incidence $\mathrm{x}$-ray standing waves. Phys. Rev. B. 74, 035404 (2006).

33. Wang, T. \& Chan, W.-L. Dynamical localization limiting the coherent transport range of excitons in organic crystals. J. Phys. Chem. Lett. 5, 1812-1818 (2014).

34. Pimpinelli, A. \& Villain, J. Physics of crystal growth. (Cambridge university press, 1998).

35. Gopakumar, T. G. et al. Adsorption of palladium phthalocyanine on graphite: STM and LEED study. J. Phys. Chem. B. 108, 7839-7843 (2004).

36. Scheffler, M. et al. Structural study of monolayer cobalt phthalocyanine adsorbed on graphite. Surf. Sci. 608, 55-60 (2013).

37. Xie, W., Xu, J., An, J. \& Xue, K. Correlation between molecular packing and surface potential at vanadyl phthalocyanine/HOPG interface. J. Phys. Chem. C. 114, 19044-19047 (2010).

38. Wang, T. et al. Effect of interlayer coupling on ultrafast charge transfer from semiconducting molecules to mono-and bilayer graphene. Phys. Rev. Applied 4, 014016 (2015).

39. Wang, L., Chen, W. \& Wee, A. T. S. Charge transfer across the molecule/metal interface using the core hole clock technique. Surf. Sci. Rep. 63, 465-486 (2008).

40. Demetrio Filho, A. \& Neto, P. H. O. Intramolecular reorganization energy in zinc phthalocyanine and its fluorinated derivatives: a joint experimental and theoretical study. Chem. Commun. 49, 6069-6071 (2013).

41. Bredas, J. L., Beljonne, D., Coropceanu, V. \& Cornil, J. Charge-Transfer and Energy-Transfer Processes in $\pi$-Conjugated Oligomers and Polymers: a Molecular Picture. Chem. Rev. 104, 4971-5004, doi: 10.1021/cr040084k (2004).

42. Lindstrom, C. D. \& Zhu, X.-Y. Photoinduced electron transfer at molecule-metal interfaces. Chem. Rev. 106, 4281-4300 (2006).

43. Partoens, B. \& Peeters, F. M. From graphene to graphite: electronic structure around the K point. Phys. Rev. B. 74, 075404 (2006).

44. Wei, C. et al. Probing the ultrafast electron transfer at the $\mathrm{CuPc} / \mathrm{Au}(111)$ interface. Appl. Phys. Lett. 88 (2006).

45. Dou, W. et al. Investigation on the orderly growth of thick zinc phthalocyanine films on Ag (100) surface. J. Chem. Phys. 133, 144704 (2010).

46. Echenique, P. M. et al. Decay of electronic excitations at metal surfaces. Surf. Sci. Rep. 52, 219-317 (2004).

47. Chizhov, I., Scoles, G. \& Kahn, A. The influence of steps on the orientation of copper phthalocyanine monolayers on Au (111). Langmuir 16, 4358-4361 (2000).

48. Ludwig, C. et al. Epitaxy and scanning tunneling microscopy image contrast of copper-phthalocyanine on graphite and MoS 2 . J. Vac. Sci. Technol. B. 12, 1963-1966 (1994).

49. Liu, Q. et al. Metal-catalyst-free and controllable growth of high-quality monolayer and AB-stacked bilayer graphene on silicon dioxide. Carbon 96, 203-211 (2016).

50. Li, X. et al. Transfer of large-area graphene films for high-performance transparent conductive electrodes. Nano Lett. 9, 4359-4363 (2009).

\section{Acknowledgements}

The work is primarily supported by US National Science Foundation, grant DMR-1351716. W.-L.C. also acknowledges the startup fund and new faculty general research fund provided by University of Kansas. J.W. acknowledges support of ARO W911NF-16-1-0029. The AFM measurement was done at the KU Bioengineering Research Center.

\section{Author Contributions}

T.W. and W.-L.C. designed the research; T.W., T.R.K. and B.K. performed the photoemission and AFM experiments; T.W. analyzed the data; T.W. and T.R.K. prepared the organic thin films; Q.L. prepared the graphene sample and is supervised by J.W.; the paper is written by T.W. and W.-L.C. and all authors commented on the paper; W.-L.C. supervised the research.

Additional Information

Supplementary information accompanies this paper at http://www.nature.com/srep

Competing financial interests: The authors declare no competing financial interests.

How to cite this article: Wang, T. et al. Growing Ultra-flat Organic Films on Graphene with a Face-on Stacking via Moderate Molecule-Substrate Interaction. Sci. Rep. 6, 28895; doi: 10.1038/srep28895 (2016).

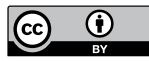

This work is licensed under a Creative Commons Attribution 4.0 International License. The images

or other third party material in this article are included in the article's Creative Commons license, unless indicated otherwise in the credit line; if the material is not included under the Creative Commons license, users will need to obtain permission from the license holder to reproduce the material. To view a copy of this license, visit http://creativecommons.org/licenses/by/4.0/ 